\title{
Kieran C. Murphy, Peter J. Scambler (eds): Velo-cardio-facial syndrome: a model for understanding microdeletion disorders
}

\section{Cambridge University Press, Cambridge, 2005, ISBN 0-521-82185-1}

Published online: 9 November 2005

(C) The Japan Society of Human Genetics and Springer-Verlag 2005
Genetics- and etiology-minded physicians with a keen observant eye may differentiate rarity from commonality in clinical practice, despite only subtle changes in phenotypic features. When a center for a specific disease is established for treatment or research purposes, patients with the relevant morbid condition will be referred to specialists working at the center. With time, the patient load will increase, and out of the group will emerge rare cases with characteristic features that attract the attention of leading specialists. Thus begins a search for a new disease entity or to find an underlying pathological mechanism, eventually opening new fields of study. At present, human features (phenotypes) are roughly categorized genetically into one of several groups, including single gene anomalies, polygenic environment-geneinteractions, chromosomal aberrations, microdeletions, as well as mitochondrially inherited conditions and uniparental dysomy or imprinting. Epigenetic and environmental factors also play important roles in causation.

The fascinating story of the discovery and understanding of the microdeletion-associated condition known as Velo-Cardio-Facial Syndrome (VCFS), from the first independent encounters by investigators in different disciplines, in different countries, and with different clinical backgrounds, has been well described in a historical overview by Shprintzen [Shprintzen et al (1978) Cleft Palate J 15:56-62], who discovered and named VCFS. The story can be summarized as follows: in the 1960s-1970s, children with a nasal voice, speech disorders and learning disability or behavioral problems were referred to a center for craniofacial and speech disorder at a hospital in New York City. These patients, who had a cleft lip or palate (submucous cleft palate)

\footnotetext{
A. Takao

Japan Research Promotion Society for Cardiovascular Disease, Tokyo Women's Medical University, 8-1 Kawada-cho, Shinjuku-ku, Tokyo 162-8666, Japan

E-mail: czw07654@nifty.com
}

and a right aortic arch or conotruncal heart defect, presented with characteristic facial features forming a syndrome that Shprintzen aptly called Velo-Cardio-Facial Syndrome. Takao, a pediatric cardiologist at the Heart Institute of Japan in Tokyo was kept busy taking care of blue babies and children with conditions such as tetralogy, pulmonary atresia, truncus, vascular anomalies, etc. Among them was a subset of children with hypernasality of voice, and characteristic facial features [Kinouchi et al (1976) Pediatr Jpn 17:84-87]. These features were so specific for the group that the name Conotruncal Anomaly Face Syndrome (CAFS) was proposed to attract the attention of pediatricians and cardiologists in Japan. Several infants with this syndrome had hypocalcemic seizures and thymic hypoplasia. In 1965, in a discussion of a colleague's paper relating to immune disorders in children, Angelo DiGeorge described athymia and immune disorder in a child with a right aortic arch, and further experience of a series of cases expanded the phenotype to include conotruncal heart anomalies in the birth defect literature [DiGeorge (1965) J Pedatr 67(Suppl):907-909]. Furthermore, in both VCFS and CAFS, it was noticed that a relatively high prevalence of schizophrenia became a real clinical problem for the patients and their families.

The above three syndromes or sequences were described independently by clinicians and investigators in different disciplines. However, through the steady efforts made toward elucidation of the pathogenetic mechanisms, especially with progress in molecular cytogenetic analysis, it was found that all three syndromic phenotypes are associated with deletions encompassing genes mapped to 22q11. VCFS is a genetic disorder associated with a deletion of the long arm of chromosome 22, which is the most common interstitial deletion disorder found in man, affecting every major system in the body with more than 100 physical and behavioral phenotypic features.

Interstitial deletions occur within chromosomal segments between the centromere and the telomeres. Im- 
proved banding and fluorescent in situ hybridization (FISH) techniques allow us to detect interstitial microdeletions that are too small to be visualized by routine karyotyping. These usually encompass multiple genes (in the case of VCFS, $>30$ due to a deletion of $1.5-3 \mathrm{Mb}$ ), causing aggregate phenotypes, which form syndromes expressing the sum of phenotypes expected from the haplo-insufficiency of genes within the deletion region.

Velo-cardio-facial syndrome: a model for understanding microdeletion disorders summarizes recent progress in the understanding and treatment of VCFS. The focus is on clinical issues, with chapters devoted to psychiatric disorders, neuroimaging, speech and language disorders, as well as to cardiac, ENT, gastrointestinal, ophthalmic, and urological manifestations. Molecular genetics, immunodeficiency, and genetic counselling are also dis- cussed. Professionals studying or treating one aspect of this deletion syndrome may be unaware of the involvement of other systems - this book will assist them in obtaining a more holistic view of patients with VCFS (del 22q11.2). A phenotypic aggregate indicates the need for a multi-disciplinary holistic approach and for lifelong follow-up of patients, during which new sequelae may appear. Microdeletion syndromes (VCFS, Williams, Alagille and others) represent one aspect of diversified human phenotypes in evolutionary history. VCFS may be seen as a paradigm for other less common microdeletion disorders, and experience with this syndrome may be most informative and thus help to direct research and treatment strategies. Clinicians and scientists of different disciplines will all enjoy and benefit from reading this book. 